\title{
Co-Secure Set Domination in Graphs
}

\author{
D. Bhuvaneswari, S. Meenakshi
}

\begin{abstract}
Throughout this paper, consider $G=(V, E)$ as a connected graph. A subset $D$ of $V(G)$ is a set dominating set of $G$ if for every $M \subseteq V / D$ there exists a non-empty set $N$ of $D$ such that the induced sub graph $\langle M U N\rangle$ is connected. A subset $D$ of the vertex set of a graph $G$ is called a co-secure dominating set of a graph if $D$ is a dominating set, and for each $u^{\prime} \in D$ there exists a vertex $v^{\prime} \in V / D$ such that $u^{\prime} v^{\prime}$ is an edge and $\left(D \backslash\left\{u^{\prime}\right\}\right) \cup\left\{v^{\prime}\right\}$ is a dominating set. A co-secure dominating set $D$ is a co-secure set dominating set of $G$ if $D$ is also a set dominating set of $G$. The co-secure set domination number $\gamma_{c s}^{s}(G)$ is the minimum cardinality of a co-secure set dominating set. In this paper we initiate the study of this new parameter \& also determine the co-secure set domination number of some standard graphs and obtain its bounds.
\end{abstract}

Keywords: Co-secure dominating set, Dominating set, Set dominating set.

\section{INTRODUCTION}

Let $\mathrm{G}=(\mathrm{V}, \mathrm{E})$ be a connected, finite, undirected graph without loops and multiple edges. The order and size of the graph $\mathrm{G}$ is $\mathrm{m}$ and $\mathrm{n}$ respectively. For $v \in G$, the number of incident edges in $\mathrm{v}$ is the degree of a vertex $\mathrm{v}$ and is written as $\mathrm{d}(\mathrm{v})$ or $\operatorname{deg}(\mathrm{v})$. The $N_{G}(v)$ is an open neighborhood of a vertex $v \in V$ of a graph $\mathrm{G}$ is defined as $N_{G}(v)=\{u \in V \backslash u$ is adjacent to $v\}$ and $N_{G}[v]=N_{G}(v) \cup\{v\}$ is a closed neighborhood of a graph G. For a set, $I \subseteq V$, $N_{G}(I)=\bigcup_{v \in I} N_{G}(v)$ and $N_{G}[I]=N_{G}(I) \cup I \quad$ is a closed neighborhood of a graph $\mathrm{G}$.

The path of $\mathrm{m}$ vertices is denoted by $P_{m}$ and cycle of $\mathrm{m}$ vertices is denoted as $C_{m}$. The complete graph of $\mathrm{m}$ vertices is a graph having every vertices of degree m-1 and is denoted by $K_{m}$. The wheel of $\mathrm{m}$ vertices is a graph obtained by joining all the vertices of cycle $C_{m-1}$ to the vertex at the centre of the cycle and is denoted by $W_{m}, m \geq 4$. The $K_{r, s}$ is a complete Bipartite graph with the partite sets $\mathrm{R}$ and $\mathrm{S}$ having cardinality $\mathrm{r}$ and $\mathrm{s}$ respectively. The graph $K_{1, m}$ is star[4],[5]. The Friendship graph $F_{m}$ is also a graph with $2 \mathrm{~m}+1$ vertices having $\mathrm{m}$ copies of $K_{3}$ with central vertex of degree $2 \mathrm{~m}$ and all other vertices of degree 2 .

Revised Manuscript Received on December 5, 2019.

D. Bhuvaneswari, Research Scholar, Department of Mathematics, Vels Institute of Science, Technology \& Advanced Studies, Chennai-600117, Tamil Nadu, India. Email: bhuvanamaths15@gmail.com.

S. Meenakshi, Associate Professor, Department of Mathematics, Vels Institute of Science, Technology \& Advanced Studies, Chennai-600117, Tamil Nadu, India. Email: meenakshikarthikeyan@yahoo.co.in
A subset $\mathrm{D}$ of $\mathrm{V}(\mathrm{G})$ is a dominating set of $\mathrm{G}$ if for every vertex $u^{\prime} \in V \backslash D$ there exist atleast one vertex $v^{\prime} \in D$ such that $u^{\prime}$ is adjacent to $v^{\prime}$. The domination number of a graph is denoted by $\gamma(G)$ which is minimum cardinality of a dominating set of $\mathrm{G}[4]$,[5].

For any connected graph $G$, a subset $D$ of $V(G)$ is a set dominating set of $\mathrm{G}$ if for every $\mathrm{M} \subseteq \mathrm{V} / \mathrm{D}$ there exists a non-empty set $\mathrm{N} \subseteq \mathrm{D}$ such that the sub graph <MUN> induced by MUN is connected and its minimum cardinality of a set dominating set of $\mathrm{G}$ is the set domination number and is denoted by $\gamma_{s}(G)$ and is abbreviated as a SD-set (set dominating set)[6].

For any non-trivial graphs $\mathrm{G}$ with a subset $\mathrm{D}$ of the vertex set $\mathrm{V}(\mathrm{G})$ is a co-secure dominating set of a graph $\mathrm{G}$ if $\mathrm{D}$ is a dominating set and for every $u^{\prime} \in D$ there exists a vertex $v^{\prime} \in V \backslash D$ such that $u^{\prime} v^{\prime}$ is an edge and $\left(D \backslash\left\{u^{\prime}\right\}\right) \cup\left\{v^{\prime}\right\}$ is a dominating set[1]. The minimum cardinality of a co-secure dominating set (or CSDS) of $\mathrm{G}$ is the co-secure domination number of $\mathrm{G}$ and its denoted by $\gamma_{c s}(G)[1]$.

The concept of co-secure domination in graphs was introduced and the co-secure domination number of path and a cycle was determined by Arumugan S., KaramEbadi and Martin Manrique [1]. Aleena Joseph and Sangeetha determined the co-secure domination number of Friendship graph, Jahangir graph and Helm graph and also obtained the bounds[2]. For any graph $\mathrm{G}$ with isolated vertices, there will be no CSDS for $\mathrm{G}$.

We introduce a new variant of co-secure domination namely the co-secure set domination set( abbreviated as CSSDS) and we initiate the study of CSSD set. A co-secure dominating set $\mathrm{D}$ is a co-secure set dominating set of $\mathrm{G}$ if $\mathrm{D}$ is also a set dominating set of $\mathrm{G}$. The minimum cardinality of CSSD set is the co-secure set domination number and is denoted as a $\gamma_{c S}^{s}(G)$. Throughout this paper, we consider the finite, connected, nontrivial graphs and undefined terms are in[4],[5].

We need the following result in[1].

\section{Corollary 1.1}

For every integer $n \geq 3, \gamma\left(P_{n}\right)=\gamma\left(C_{n}\right)=\left\lceil\frac{n}{3}\right\rceil$.

\section{MAIN RESULT}

In this Section, we analyze the new parameter called the co-secure set domination number of various other graphs such as Path graph, Cycle graph, Complete graph, Wheel graph, Complete Bipartite graph, Star graph and Friendship graph. Moreover, we obtain the bounds for co-secure set domination number for any 


\section{Co-secure Set Domination in Graphs}

nontrivial graph with order $\mathrm{m}$.

\section{A. Definition}

Let $\mathrm{G}=(\mathrm{V}, \mathrm{E})$ be any non-trivial graph. A Co-secure dominating set of $\mathrm{G}$ is called a Co-secure set dominating set if for every $\mathrm{M} \subseteq \mathrm{V} / \mathrm{D}$ there exists a set $\mathrm{N} \subseteq \mathrm{D}$ which is non empty such that the sub graph $<$ MUN $>$ induced by MUN is connected. The minimum cardinality of co-secure set dominating set of $\mathrm{G}$ is defined as the co-secure set domination number of $\mathrm{G}$ and is denoted as $\gamma_{s}^{c s}(G)$.

From this, we say that a dominating set $\mathrm{D}$ is a co-secure set dominating set if and only if $\mathrm{D}$ is co-secure as well as set dominating set.

\section{B. Observation}

$$
\text { If } \Delta(\mathrm{G})=\mathrm{m}-1 \text {, then } \gamma(G) \leq \gamma_{s}(G) \leq \gamma^{c s}(G) \leq \gamma_{s}^{c s}(G) \text {. }
$$

\section{Observation}

For a Path $\mathrm{P}_{\mathrm{m}}, \gamma_{s}^{c s}\left(P_{m}\right)=\left\{\begin{array}{c}m-1, \text { for } \mathrm{m}=2,3, \\ m-2, \text { for } m=4,5,6, \\ \text { does not exist, } \text { for } \mathrm{m} \geq 7\end{array}\right.$

\section{Observation}

If $\mathrm{G}$ is a cycle graph of $\mathrm{m}$ vertices, then $\gamma_{s}^{c s}\left(C_{m}\right)=\left\{\begin{array}{c}m-2, \quad \text { for } \mathrm{m}=3,4, \\ m-3, \text { for } 5 \leq \mathrm{m} \leq 9, \\ \text { does not exist, } \text { for } \mathrm{m} \geq 10\end{array}\right.$

\section{E. Theorem}

If $\mathrm{G}$ is a complete graph with $\mathrm{m}$ vertices, then $\gamma_{s}^{c s}\left(K_{m}\right)=1$ for $m \geq 3$.

\section{Proof}

Let $G=K_{m}$ be a graph with $\mathrm{m}$ vertices each of degree $\mathrm{m}-1$, then we have to prove that $|S|=1$ is a minimum co-secure set dominating set. Instead of this, it is enough to show that $\mathrm{S}$ is a minimum dominating set of $K_{m}$ because every vertex is of degree m-1. Thus every vertex of $K_{m}$ is connected to every other vertex of that graph. So minimum dominating set of $K_{m}$ is 1 . Therefore, $\gamma_{s}^{c s}\left(K_{m}\right)=1$.

\section{F. Theorem}

If $W_{m}$ is a wheel graph with $m$ vertices then $\gamma_{s}^{c s}\left(W_{m}\right)=\left\lceil\frac{m-1}{3}\right\rceil$ for $m \geq 4$.

\section{Proof}

Let $W_{m}=C_{m-1}+\{v\}$, where $C_{m-1}$ is a cycle of order m-1. Let $\mathrm{S}$ be any CSSDS. If $\{v\}$ is not in $\mathrm{S}$, then $\mathrm{S}$ is a co-secure set dominating set of $C_{m-1}$ and hence $|S| \geq\left\lceil\frac{m-1}{3}\right\rceil$ for $6 \leq m \leq 10$ and no such $\mathrm{S}$ exists for $m \geq 11$ because the set $\mathrm{S}$ is having the occurrence of consecutive vertices continuously 3 or more than so $S$ will be a set dominating set but that $S$ will not be co-secure dominating set. If $\{v\} \in S$, then $S$ will be

considered as a dominating set because $\mathrm{v}$ is connected with all the vertices of $C_{m-1}$ and there exist $u \in V\left(C_{m-1}\right)$ such that $\mathrm{u}$ replaces v. Since $C_{m-1}-N[u]=P_{n-4}$ it follows from the corollary 1.1 that $|S| \geq\left\lceil\frac{m-4}{3}\right\rceil+1=\left\lceil\frac{m-1}{3}\right\rceil$. Thus $\gamma_{s}^{c s}\left(W_{m}\right) \geq\left\lceil\frac{m-1}{3}\right\rceil$. Further any $\gamma-$ set of $C_{m-1}$ is an CSSDS of $W_{m}$ (i.e. $\gamma$-set of $\left.\left(C_{m-1} \backslash\{u\}\right) \cup\{v\}\right)$, gives that $\gamma_{s}^{c s}\left(W_{m}\right) \leq\left\lceil\frac{m-1}{3}\right\rceil$. Hence, $\gamma_{s}^{c s}\left(W_{m}\right)=\left\lceil\frac{m-1}{3}\right\rceil$ for $m \geq 4$.

\section{Remark}

Also for $m \geq 4, \gamma^{c s}\left(W_{m}\right)=\left\lceil\frac{m-1}{3}\right\rceil$ is already found in [1].

Thus we have $\gamma^{c s}\left(W_{m}\right)=\gamma_{s}^{c s}\left(W_{m}\right)=\left\lceil\frac{m-1}{3}\right\rceil$.

\section{G. Theorem}

For a graph $G=K_{1, m-1}$, then $\gamma_{s}^{c s}\left(K_{1, m-1}\right)=m-1$.

\section{Proof}

Let $G=K_{1, m-1}$, then we say that $\mathrm{S}$, the set of all pendant vertex of $K_{1, m-1}$ is a minimum co-secure set dominating set because the central vertex must always be in V\S, then only for every vertex $\mathrm{v}_{\mathrm{i}}$ in $\mathrm{S}$ there exists a vertex $\mathrm{v}$ in $\mathrm{V} \backslash \mathrm{S}$ such that $\mathrm{vv}_{\mathrm{i}}$ is an edge of $K_{1, m-1}$ and $\left(\left(S \backslash\left\{v_{i}\right\}\right) \cup\{v\}\right)$ will form a dominating set of $K_{1, m-1}$ and therefore $|S|=m-1$. Hence $\gamma_{s}^{c s}\left(K_{1, m-1}\right)=m-1$.

\section{H. Theorem}

For a Complete Bipartite graph $K_{r, s}$ with $r \leq s$, then $\gamma_{s}^{c s}\left(K_{r, s}\right)= \begin{cases}s, & \text { if } r=1, \\ 2, & \text { if } r=2, \\ 3, & \text { if } r=3, \\ 4, & \text { if } r \geq 4,\end{cases}$

\section{Proof}

Let $R=\left\{u_{1}, u_{2}, u_{3}, \ldots u_{r}\right\}$ and $S=\left\{u_{1}^{\prime}, u_{2}^{\prime}, u_{3}^{\prime}, \ldots u_{s}^{\prime}\right\}$ be the bipartition of $G=K_{r, s}$. If $\mathrm{r}=1$ then $G=K_{1, s}$ is a star and the proof is given in the theorem 2.7. If $\mathrm{r}=2$, then $\gamma_{s}^{c s}\left(K_{2, s}\right)=2$. If $\mathrm{r}=3$ and let $S_{1}$ be a CSSDS of $K_{3, s}$. If $S_{1} \cap R=\left\{u_{1}\right\}$ and $S_{1} \cap S=\left\{u_{1}^{\prime}\right\}$, then no vertex in R or $\mathrm{S}$ can replace $u_{1}$ or $u_{1}^{\prime}$. Therefore $\gamma_{s}^{c s}\left(K_{3, s}\right) \geq 3$. Also $\mathrm{R}$ is a CSSDS of $K_{3, s}$ and so $\gamma_{s}^{c s}\left(K_{3, s}\right)=3$. Now, if $r \geq 4$ and let $S_{1}$ be a CSSDS of $K_{r, s}$. We know that if $S_{1} \cap R=\varnothing$, then $S_{1}=S$ and vice versa. Further if $S_{1} \cap R \neq \varnothing$, then $\left|S_{1} \cap R\right| \geq 2$ and if $S_{1} \cap S \neq \varnothing$, then $\left|S_{1} \cap S\right| \geq 2$. So in this case $\left|S_{1}\right| \geq 4$. Also if $\left\{u_{1}, u_{2}, u_{1}^{\prime}, u_{2}^{\prime}\right\}$ is a CSSDS of 
$K_{r, s}$ and hence we conclude that $\left|S_{1}\right|=4$. Therefore $\gamma_{s}^{c s}\left(K_{r, s}\right)=4$ for $r \geq 4$.

\section{Theorem}

Let $F_{m}$ be a Friendship graph having $2 \mathrm{~m}+1$ vertices, then $\gamma_{s}^{c s}\left(F_{m}\right)=m$ for all $\mathrm{m}$.

\section{Proof}

Let $F_{m}$ be a friendship graph with $2 \mathrm{~m}+1$ vertices such that $F_{m}$ consists of m copies of $K_{3}$. Let the central vertex of $F_{m}$ be $\mathrm{u}$ and its degree is $2 \mathrm{~m}$ and $v_{1}, v_{2}, v_{3} \ldots . . v_{2 k}$ be the vertices labeled in clockwise direction (or anticlockwise) with each vertex $v_{i}$ having degree 2 .

If $\{u\}$ is not in $S$, then every set in V $\backslash S$ is not connected to any set in $\mathrm{S}$. Therefore, there is no such CSSDS for this case. If $\{u\} \in S$, and also choose one vertex from each m-1 copies of $K_{3}$ and form the CSSDS with $\mathrm{m}$ vertices. That is, $S=\left\{v_{1}, v_{3}, v_{5}, \ldots . . v_{2 m-3}, u\right\}$ $S_{1}=\left\{v_{2}, v_{4}, v_{6}, \ldots \ldots v_{2 m-2}, u\right\}$ be a CSSDS of $F_{m}$. For this $\mathrm{S}$ (or $\mathrm{S}_{1)}$, every set in $\mathrm{V} \backslash \mathrm{S}$ is connected to any set in $\mathrm{S}$ and also $\left(S \backslash\left\{v_{i}\right\}\right) \cup\left\{v_{i+1}\right\}, \quad(S \backslash\{u\}) \cup\left\{v_{2 m-1}\right\}$ for $i=1,3,5, \ldots 2 m-3$ forms a dominating set (or $\left(S_{1} \backslash\left\{v_{i}\right\}\right) \cup\left\{v_{i-1}\right\}, \quad\left(S_{1} \backslash\{u\}\right) \cup\left\{v_{2 m}\right\} \quad$ for $\quad$ all $i=2,4,6, \ldots 2 m-2$ forms a dominating set). Thus $\gamma_{s}^{c s}\left(F_{m}\right) \leq m$.

Now we have to verify that $\mathrm{S}$ is the minimum CSSDS . That is, there exist no CSSDS with less than $m$ vertices. Let us assume that $\gamma_{s}^{c s}\left(F_{m}\right)<m$. But at most one vertex from each $\mathrm{m}-1$ copies of $\mathrm{K}_{3}$ and central vertex $u$ must be in the CSSDS (otherwise it's not a CSSDS), which is a contradiction.

Therefore, $\gamma_{s}^{c s}\left(F_{m}\right)=m$, for all $\mathrm{m}$.

\section{Remark}

Also $\gamma^{s}\left(F_{m}\right)=\gamma^{c s}\left(F_{m}\right)=m$ is already found in [2]. Thus we have $\gamma^{s}\left(F_{m}\right)=\gamma^{c s}\left(F_{m}\right)=\gamma_{s}^{c s}\left(F_{m}\right)=m$.

\section{J. Theorem}

For any graph $\mathrm{G}$ of order $\mathrm{m}$ without isolated vertex, then $1 \leq \gamma_{s}^{c s}(G) \leq m-1$. Furthermore, the $\gamma_{s}^{c s}(G)=1$ if and only if $G=K_{m}$ and the equality holds in the upper bound, $\gamma_{s}^{c s}(G)=m-1$ if and only if $G=K_{1, m-1}$.

\section{Proof}

The bounds are trivial. Now consider the lower bound. The result is obvious if $G=K_{m}$, then $\gamma_{s}^{c s}(G)=1$. Conversely, if there is a co-secure set dominating set, $S$ with $|S|=1$, i.e., $S=\left\{u_{1}\right\}$ and that $u_{1}$ dominates every other vertex in V $\backslash \mathrm{S}$, therefore $\operatorname{deg} u_{1}=m-1$. Also every set in $\mathrm{V} \backslash \mathrm{S}$ is connected with any set in S. Since $S=\left\{u_{1}\right\}$ is also a co-secure dominating set, so if we replace $u_{1}$ by any other vertex $\left\{u_{i}\right\}$ in $\mathrm{V} \backslash \mathrm{S}$ for $i=2,3,4, \ldots . . m$ is also a dominating set. From this we conclude that every vertex in $\mathrm{V} \backslash \mathrm{S}$ is also adjacent to every other vertex of $\mathrm{G}$. Therefore $\operatorname{deg} u_{i}=m-1$ for $i=2,3,4, \ldots . . m$. Hence $G=K_{m}$.

Now consider the upper bound.

Suppose $G=K_{1, m-1}$, then $\gamma_{s}^{c s}(G)=m-1$ is given in the theorem 2.7. Conversely, suppose $\gamma_{s}^{c s}(G)=m-1$. Let $\mathrm{S}$ be a minimum co-secure set dominating set with $|S|=m-1$ and let $V \backslash S=\{v\}$. Then all the vertices in $\mathrm{S}$ are adjacent only to v. If not, then there exists a set $\left\{v_{1}, v_{2}\right\} \subseteq S$ such that $v_{1} v_{2}$ is an edge of $K_{1, m-1}$, and also $S \backslash\left\{v_{1}\right\}$ is a co-secure set dominating set of cardinality less than $\mathrm{m}-1$, a contradiction. Therefore, all the vertices in $\mathrm{S}$ are leaves of $\mathrm{G}$ (i.e., vertices in $\mathrm{S}$ are independent). Hence we conclude that $G=K_{1, m-1}$.

\section{CONCLUSION}

We have a new variant of the co-secure domination namely co-secure set domination set and co-secure domination number of various graph such as path, cycle, complete graph, wheel graph, star graph, complete bipartite graph and friendship graph and also its bounds. We observed for the wheel, complete, complete bipartite, star and friendship graph have $\gamma_{s}^{c s}(G)=\gamma^{c s}(G)$. In this paper we get the co-secure set domination number is always less than its order. What will happen to $\gamma_{s}^{c s}(G)$ if we remove or add a vertex to a graph $\mathrm{G}$ is an open area for investigation.

\section{REFERENCES}

1. S. Arumugam, Karam Embadi, Martin Manrique, "Co-secure and Secure Domination in Graphs," Utilias Mathmatica, 94, 2014, pp.167-182.

2. Aleena Joseph, V. Sangeetha, "Bounds on Co-secure Domination in Graphs", International Journal of Mathematics Trends and Technology (IJMTT), vol.5, 2018, pp. 158-164

3. E.J. Cockayne, P.J.P. Grobler, W.R. Grundlingh, J. Munganga, J. H. Van Vuuren, "Protection of Graph," Utilias Mathematica, vol.67, 2005 pp.19-32.

4. T.W. Haynes, S.T. Hedetniemi, and P.J.B Slater, Fundamentals of Domination in Graphs, Marcel Dekker, Inc. New York, 1998.

5. T.W. Haynes, S.T. Hedetniemi, and P.J.B Slater, Domination in Graphs. Advanced Topics, Marcel Dekker, Inc. New York, 1998.

6. E. Sampathkumar, and PusphaLatha, "Set domination in graphs," $J$ Graph theory, vol. 18, no. 5, 1994, pp. 489-495. 